Euskal ikerketen aldizkaria | Revue d'études basques |

Revista de estudios vascos | Basque studies review

$12 \mid 2008$

Numéro XII

\title{
A [texte intégral]
}

A.B. Barne aldizkaria, A.B. Saison, AC ! Pays basque, A.C.B.B. Anglet-Côte basque-basket, A.D.I.L.

\section{(2) OpenEdition}

1 Journals

\section{Édition électronique}

URL : http://journals.openedition.org/lapurdum/1642

DOI : 10.4000/lapurdum. 1642

ISSN : 1965-0655

Éditeur

IKER

\section{Édition imprimée}

Date de publication : 1 février 2008

Pagination : 17-34

ISBN : 978-2-86781-617-8

ISSN : $1273-3830$

\section{Référence électronique}

《A [texte intégral] », Lapurdum [En ligne], 12 | 2008, mis en ligne le 24 mai 2010, consulté le 30 avril 2019. URL : http://journals.openedition.org/lapurdum/1642 ; DOI : 10.4000/lapurdum.1642

Ce document a été généré automatiquement le 30 avril 2019

Poitelon J.C. |IKER 


\section{A [texte intégral]}

A.B. Barne aldizkaria, A.B. Saison, AC ! Pays basque, A.C.B.B. Anglet-Côte basque-basket, A.D.I.L.

1 A.B. Barne aldizkaria. $1999-2000\left(3 \mathrm{n}^{\circ}\right)$ [?]. - Baiona, $21 \times 15 \mathrm{~cm}$

Avec un $n^{\circ} 0$ de [déc. 1997] paru sous le titre: Abertzaleen batasunaren barne aldizkaria. - En basque et français. - Publ. par : Abertzaleen batasuna [Bayonne]

2 A.B. Saison voir Aviron bayonnais, club omnisports. Saison

3 AC! Pays basque. Agir ensemble contre le chômage. Bulletin d'information de l'association Agir ensemble contre le chômage. déc. 1998 ( $\left.{ }^{\circ} 1\right)$. - [Bayonne], $30 \times 21 \mathrm{~cm}$ En français

4 A.C.B.B. Anglet-Côte basque-basket. Saison. Annuel. 2001/02 $\rightarrow$. Anglet, $30 \times 21 \mathrm{~cm}$ En français. - Suite de : Réveil amical sportif d'Anglet...

A.D.I.L. voir Association de défense des intérêts de Lahonce

6 A.D.N. Pays basque. La Soldanella villosa voir Soldanella (La) villosa

7 A.M.M.A.C. Bayonne.Amicale des anciens marins de Bayonne. Annuel. 1990-2000/01 ( ${ }^{\circ}$ 1-14). - Bayonne, $30 \times 21 \mathrm{~cm}$

Jusqu'en $1992\left(n^{\circ} 5\right)$ a paru sous le titre: Amicale des anciens marins et marins anciens combattants de Bayonne. - En français. - Publ. par : Amicale des anciens marins et marins anciens combattants de Bayonne [Bayonne]

8 A.P.E.L. Saint-Thomas d'Aquin info. 2 ou $3 n^{\circ}$ par an puis irrégulier. 1984 [?] $\rightarrow$. Saint-Jeande-Luz, $30 \times 21 \mathrm{~cm}$

En mai 2002 a paru sous le titre: Saint-Thomas actua; en 2003 a paru sous le titre: SaintThomas info. - En français. - Publ. par : Association des parents d'élèves de l'enseignement libre de Saint-Thomas d'Aquin [Saint-Jean-de-Luz]

9 A.R.S.I.P. \{Association de recherches spéléologiques internationales de la Pierre-SaintMartin\}. Annuel puis irrégulier. 1966 - 1974/76 (n¹-9/11). 1977/1980 (n¹2/15). [1981/1988] (n'16). - Arette, $30 \times 21 \mathrm{~cm}$

En 1977/1980 titre de départ : Bulletin de l'A.R.S.I.P. - En français. - Publ. par : Association de recherches spéléologiques internationales de la Pierre-Saint-Martin puis Association pour 
la recherche spéléologique internationale à la Pierre-Saint-Martin [Sainte-Engrâce]. - Les n¹- 9/11 ont donné lieu à une rééd. en 1981

10 A.R.S.I.P.-info. Irrégulier. $1975 \rightarrow$. Arette puis Monein, $30 \times 21 \mathrm{~cm}$

En français. - Publ. par : Association de recherches spéléologiques internationales de la Pierre-Saint-Martin puis Association pour la recherche spéléologique internationale à la Pierre-Saint-Martin [Sainte-Engrâce]

11 A.S.B. Panorama sportif voir Association sportive bayonnaise. Saison...

12 A l'affiche. Bayonne, Anglet, Biarritz, Boucau [ou Anglet, Bayonne, Biarritz, \{Boucau\}]. Arts, culture, loisirs [puis Votre agenda culturel]. Bimestriel. mai/juin 1996 - sept./oct. 1997 (n¹-8). sept./oct. 1998 (n.s. nº 1 ) $\rightarrow$. Bordeaux puis Bayonne, Anglet, Biarritz, \{Boucau\}, $40 \times 28$ puis $30 \times 15 \mathrm{~cm}$

Le sous-titre varie et disparait. - En français. - Publ. par les villes citées dans le sous-titre. Jusqu'en 1997 suppl. local de : Sud-Ouest [Bordeaux]

13 A l'air libre. Théâtre du Versant. \{Le journal de la compagnie\}. Trimestriel. oct./déc. 1997 $\left[\mathrm{n}^{\circ} 1\right] \rightarrow$. Biarritz, $30 \times 21 \mathrm{~cm}$

En français. - Jusqu'en avr./juin 1998 paraît avec : Lézarde (La). Le trimestriel de Lézards qui bougent

A savoir voir Jakingarri

$15 *$ Aana. Mensuel. [...]. - Bayonne

Paraissait en 1946. - En basque et français. - Publ. par : Ligue féminine d'action catholique française [Bayonne]. - Cité dans : Herria le 26 sept. 1946 et dans un article de Pierre Lafitte paru en sept./oct. 1966 dans : Elgar

Abbadiako kutzukeriak voir Cancans et autres potins d'Abbadia

Abbaye N.-D. de Belloc [puis Abbaye N.-D. de Belloc. Monastère Ste Scholastique]. Lettre aux oblats. 3 ou $4 n^{\circ}$ par an. mars 1970 - déc. 2001 (n॰1-115). - Urt, $28 / 30 \times 21 \mathrm{~cm}$

Avec un $n^{\circ} 0$ du 3 janv. 1970. - Le $n^{\circ} 1$, de format $21 \times 15 \mathrm{~cm}$, a paru sous le titre : Lettre aux oblats de Belloc. - En français. - Quelques nportent : Supplément à Corde magno; voir ce titre, qui devient : Voix de Belloc

Abentura. Euskal herriko kultur ekitaldi nagusi ta herri besteetako egutegia. Principales manifestations culturelles et fêtes de village du Pays basque. juil./sept. 1999 - été/ automne $2000(2 \mathrm{n})$. - [Bayonne], $15 \times 11 \mathrm{~cm}$

En basque, espagnol et français [puis aussi anglais]. - Publ. par : Abentura [Bayonne]

Abertzaleen batasuna mintzo. [nov. 1998 - avr. 1999] (n¹-2). - Bayonne, $42 \times 29 \mathrm{~cm}$

En basque et français. - Publ. par : Abertzaleen batasuna [Bayonne]

Abertzaleen batasunaren barne aldizkaria voir A.B. Barne aldizkaria

21 Abertzaleen oihartzuna. 1992. - Impr. à Saint-Jean-Pied-de-Port, $30 \times 21 \mathrm{~cm}$

En basque et français. - Publ. à l'occasion des élections cantonales de mars 1992

Abil. \{Bulletin mensuel d'expression locale et d'information de la Soule\}. Mensuel puis bimestriel. oct. 1982 - avril/mai 1989 (n¹-66). oct. 1989 (n67). - Mauléon, $42 \times 28$ puis $45 \times 31$ puis $31 \times 22 \mathrm{~cm}$

En français; quelques textes basques. - Publ. par : Association pour le développement de l'expression locale et de l'information en Soule (A.D.E.L.I.S.) [Mauléon]. - Reprend certains éléments de : Egia. Bulletin mensuel du Groupe d'étude et d'information de la Soule. Remplacé par : Oldartzen; le $n^{\circ} 67$ contient le n'0 de ce journal 

$2000 \rightarrow$. Saint-Jean-de-Luz, $21 \times 15 \mathrm{~cm}$ En français. - Publ. par : Saint-Jean-de-Luz - Ciboure A.V.F. accueil [Saint-Jean-de-Luz]. Jusqu'en 1999 constitue une partie de : Accueils (Les) de la Côte basque

27 Accueils (Les) de la Côte basque. Bayonne, Biarritz, Anglet-Blancpignon, St-Jean-de-Luz [puis Bayonne, Biarritz, Anglet, Blancpignon, St-Jean-de-Luz-Ciboure, Hendaye puis Bayonne, Anglet, Blancpignon]. Mensuel. janv. $1977 \rightarrow$. Biarritz puis Bayonne puis Tarnos, $29 \times 21$ puis $22 \times 15 \mathrm{~cm}$

En 1977 a paru sous le titre : Côte basque accueil. - En français. - Publ. par les accueils des villes citées dans le sous-titre; ce sous-titre et le nombre des villes citées varient : Biarritz disparaît en 1990, Hendaye en 1998, Saint-Jean-de-Luz et Ciboure en 2000; pour Biarritz, à partir de 1990 voir Accueils des villes françaises. Biarritz; pour Saint-Jean-de-Luz et Ciboure, à partir de 2000 voir Accueil des villes françaises A.V.F. Saint-Jean-de-Luz - Ciboure

Accueils des villes françaises. Biarritz. Bimestriel ou trimestriel. 1990 - sept./déc. 2001. Biarritz, $21 \times 15 \mathrm{~cm}$

En français. - Publ. par : Biarritz A.V.F. accueil [Biarritz]. - Jusqu'en 1989 constitue une partie de: Accueils (Les) de la Côte basque. - Devient : Biarritz accueil

29 * Activités d'Ikas. Irrégulier. 1962 [?] - [...]. - Bayonne, $30 \times 21 \mathrm{~cm}$

Aurait cessé de paraître après la publication de quelques npuis reparu; paraissait encore en 1988. - En basque et français. - Publ. par : Ikas [Bayonne]. - Remplace : Ikas [1959]

Activités en Pays Basque. Commerce, industrie, tourisme. Revue mensuelle éditée par la Chambre de commerce de Bayonne. Mensuel ou irrégulier. mars 1950 - mars 2003 (n¹-459). - Bayonne, $27 \times 21$ puis $30 \times 21 \mathrm{~cm}$

Avec un $n^{\circ}$ spécial de déc. 1969 intitulé : Profil du bassin de l'Adour. - A partir d'avr. 1990 certains $n^{\circ}$ ont paru sous le titre : Lettre (La) d'Activités en Pays basque. - Le sous-titre varie. En français. - Publ. par: Chambre de commerce [puis de commerce et d'industrie] de Bayonne [puis de Bayonne Pays basque] [Bayonne]. - De févr. 1977 à mai/juin 1978 porte une double numérotation: $\mathrm{n}^{\circ} 287-298$ ou n.s. $\mathrm{n}^{\circ} 1-12$. - Reprend les éléments de: Bulletin de la Chambre de commerce de Bayonne [1926-1937]. - A partir de 1996 certains $n^{\circ}$ constitués par : Chambre de commerce et d'industrie de Bayonne Pays basque. Rapport d'activités. - Pour les suppl. voir Fil (Le) international et Fil (Le) vert. - Voir aussi Feuilles d'informations techniques

31 Actuel Pays Basque magazine. Mensuel d'actualités économiques et touristiques. mars nov. 1973 (n¹-9). - Biarritz, $27 \times 21 \mathrm{~cm}$

En oct. et nov. 1973 a paru sous le titre : Actuel magazine Pays basque. Mensuel d'actualité économique et touristique franco-espagnol. - En français; quelques textes espagnols. - Devient : Cap Sud 
32 Adostu. Piarres Larzabal kolegioaren ikasleen hilabetekaria. [avr. 2000] ( $\left.n^{\circ} 0\right)$. - [Ciboure], $30 \times 21 \mathrm{~cm}$

En basque. - Publ. par : Piarres Larzabal kolegioa [Ciboure]

33 Adour-avenir. $1^{\mathrm{er}}$ juin 1988 ( $\mathrm{n}^{\circ}$ spécial). - [Bayonne], $44 \times 32 \mathrm{~cm}$

En français. - Publ. à l'occasion des élections législatives de juin 1988 pour soutenir la candidature d'Alain Lamassoure

34 Adour-économie. Lettre hebdomadaire d'information économique des entreprises des pays de l'Adour (Landes, Pyrénées-Atlantiques, Hautes-Pyrénées). $1^{\mathrm{er}} / 7$ févr. - 30 mai/6 juin $1996\left(\mathrm{n}^{\circ} 1-18\right)$ [?]. - Ustaritz, $30 \times 21 \mathrm{~cm}$ En français

35 Adour-info. Journal d'information du Pays Basque et de l'Adour. Hebdomadaire. 18 oct. $15 / 21$ nov. $1985\left(\mathrm{n}^{\circ} 1-5\right)$. - Ciboure, $42 \times 31 \mathrm{~cm}$ En français; quelques textes basques

36 Aekazeta. Iparraldeko A.E.K.-ko barne aldizkaria. Irrégulier. [mars - mai 1986] (n¹-2). févr. 1990 - déc. $2003\left(n^{\circ} 1-30\right)$. - Bayonne, $31 \times 23$ puis $30 \times 21 \mathrm{~cm}$ En 1986 a paru sous le titre :Aekaseta; en déc. 2000 a paru sous le titre: Korrikazeta. - En basque et français. - Publ. par: Euskal herriko alfabetatze euskalduntze koordinakundea (A.E.K.) [Bayonne]

37 Aéro-club basque. Lien d'informations des aéro-clubs. Annuel puis irrégulier. 1973/74-1980. 1987 - 1996. - Biarritz, Toulouse puis Anglet, Toulouse, $27 \times 21$ puis $30 \times 21$ $\mathrm{cm}$

Jusqu'en 1974/75 a paru sous le titre: Revue d'informations de l'Aéro-club basque; la couv. porte alors : Lien d'informations des aéro-clubs. - En français. - Publ. par : Aéro-club basque [Biarritz puis Anglet]

38 Ager. [puis Pour un fédéralisme basque européen]. Mensuel puis bimensuel. juil. $1981-1^{\mathrm{er}}$ oct. 1996 ( $\left.{ }^{\circ} 1-302\right)$. - Bayonne, $30 \times 21 \mathrm{~cm}$

En français; quelques textes basques. - Publ. par: Association Ager [Bayonne]. - Reprend certains éléments de : Ager [1972-1973]. - Devient : Ager Iparralde

39 Ager. Analyses de groupe sur l'économie régionale [1972-1973] (n¹-3). - Mauléon, $27 \times 19$ $\mathrm{cm}$

En français. - En 1981 certains éléments repris par : Ager [Bayonne]

Ager Iparralde. Réflexions sur le passé, le présent, l'avenir. Gogoetak : atzo, egun, bihar. Mensuel. $1^{\text {er }}$ févr. $1997\left(n^{\circ} 1\right) \rightarrow$. Bayonne puis Louhossoa, $30 \times 21 \mathrm{~cm}$ En français; quelques textes basques. - Suite de : Ager [1981-1996]

41 Agir ensemble [puis Agir ensemble, avec vous et pour vous]. Journal de l'association Ensemble d'Eguralde à d'Ibusty [puis Journal de l'opposition municipale]. avr. 1996 - déc. $1997\left(n^{\circ} 1-5\right)$. - Mouguerre, $30 \times 21 \mathrm{~cm}$ En français. - Suite de : Ensemble... d'Eguralde à d'Ibusty...

42 Agir pour Biarritz. [déc. 1982 - mars 1983] (n¹-5). - Biarritz, 43/46 × $32 \mathrm{~cm}$ En français. - Publ. à l'occasion des élections municipales de mars 1983 pour soutenir la liste «Agir pour Biarritz »

43 Agriculture et progrès. Organe de la Fédération des groupements agricoles du Pays basque [puis du Pays basque et de l'Adour maritime]. Bimestriel ou irrégulier. sept. 1956 déc. 1962/janv. 1963 (n¹-29). févr./mars 1963 - 1966. oct. 1966 - 2e trim. 1985 (n.s. n¹-70) 
[?]. avr. 1987 (n.s. $n^{\circ} 1$ ) - [...]. - Bayonne, de $33 \times 25$ à $50 \times 33 \mathrm{~cm}$

En français; jusqu'en 1962 quelques textes basques. - En 1990 remplacé par : Génétique et progrès

Agur. Bulletin de $\left\{l^{\prime}\right\}$ Eskualdunen biltzarra \{de Bordeaux\}. Bimestriel. nov. 1953 (n¹) $\rightarrow$. Bordeaux, $24 \times 16$ puis $35 \times 25 \mathrm{~cm}$

En français; quelques textes basques

Ahetzeko berriak. [puis Bulletin municipal d'information[s]]. Irrégulier. avr. $1980\left(\mathrm{n}^{\circ} 1\right) \rightarrow$. Ahetze, $30 \times 21 \mathrm{~cm}$

Titre français jusqu'en avr. 1996 : Bulletin municipal d'Ahetze. - En français; de mai 1983 à 1985 quelques textes basques. - A partir de 1996 un n'chaque année comporte une partie, parfois séparée, intitulée : Ahetze de A à Z. - A partir de 1996 parait parallèlement à : Ahetzeko berriak. Lettre municipale...

Ahetzeko berriak. Lettre municipale d'information[s]. Irrégulier. [juin 1996] $\left(\mathrm{n}^{\circ} 1\right) \rightarrow$. Ahetze, $30 \times 21 \mathrm{~cm}$

En français. - Paraît parallèlement à : Ahetzeko berriak. Bulletin municipal...

47 Aïnhoa. mars 1983 ( $\left.\mathrm{n}^{\circ} 1\right)$. - [Aïnhoa], $34 \times 25 \mathrm{~cm}$

En français. - Publ. à l'occasion des élections municipales de mars 1983 pour soutenir la «Liste d'action et de défense des intérêts communaux» à Ainhoa

Ainhoa. Bulletin d'information municipale voir Krida

Aintzina. Bulletin des communistes marxistes - léninistes de Biarritz. [sept./oct. 1978] (n $\left.{ }^{\circ} 1\right)$ - 1979. - [Biarritz], $30 \times 21 \mathrm{~cm}$

En français. - Publ. par: Parti communiste marxiste-léniniste. Cellule Maiatzaren lehena [Biarritz]

Aitzina Xiberoa. Journal édité par le Syndicat intercantonal du Pays de Soule [puis Journal édité par la Communauté de communes de Soule puis Journal de la Communauté... ] Annuel. nov. $1999\left(\mathrm{n}^{\circ} 1\right) \rightarrow$. Mauléon, $42 \times 30 \mathrm{~cm}$

En français; quelques textes basques. - N'a pas paru en 2000

51 Akilua. Arbona zain. [puis Protéger Arbonne]. Irrégulier. mars 1992 - nov. 2000 (n¹-12). oct. 2002 ( $\mathrm{n}^{\circ} 12$ [sic]). - Arbona, $32 \times 22$ puis $30 \times 21 \mathrm{~cm}$

Avec un $n^{\circ} 0$ de [nov. 1991]. - En français; quelques textes basques. - Publ. par: Arbona zain elkartea [Arbonne]

52 Albatros (L'). \{Journal de l'Association du Centre médico-psychologique Bellevue\}. Mensuel puis bimestriel. juin 1990 - juin 1991 ( $\left.n^{\circ} 1-7\right)$. - Bayonne,30 × $21 \mathrm{~cm}$

Avec un $n^{\circ}$ spécial de [juil. 1990]. - En français. - Publ. par: Association Bellevue [Bayonne]. Suite de : Entonnoir (L')

53 Aldaia. La lettre de l'Institut du logiciel et des systèmes [puis de l'E.S.T.I.A. et du campus I.D.L.S. puis de l'Ecole supérieure des technologies industrielles avancées]. Irrégulier. mai 1993 - juin 2001 (n¹-17). - Bayonne, $30 \times 21 \mathrm{~cm}$

Porte en tête: Chambre de commerce et d'industrie de Bayonne Pays basque. - En français; quelques textes anglais. - Le n ${ }^{\circ} 15$ n'a pas paru. - Voir aussi E.S.T.I.A. news

Aldiz. Donibane-Lohitzune-Ziburuko informazio aldizkaria [puis kultur aldizkaria]. [févr.] - avr. 1990 (n¹-2). - Donibane-Lohitzune, $30 \times 21 \mathrm{~cm}$

En basque. - Publ par : Euskal herriko alfabetatze euskalduntze koordinakundea (A.E.K.). Donibaneko ikasleak [Saint-Jean-de-Luz]

Alegera. [puis Journal d'information de l'A.P.E.L. Collège Sainte Marie]. Irrégulier. janv. $1995\left(n^{\circ} 1\right) \rightarrow$. Saint-Jean-de-Luz, $30 \times 21 \mathrm{~cm}$ 
En français. - Publ. par : Association des parents d'élèves de l'enseignement libre. Collège Sainte Marie [Saint-Jean-de-Luz]

Alkarrekin. Bimestriel puis irrégulier. févr. 1990 - été 1997 (n¹-20). - Baiona, $30 \times 21 \mathrm{~cm}$

En basque et français; quelques textes espagnols. - Publ. par: Eusko alkartasuna Iparralde [Bayonne]. - Devient : Alkarrekin lan

57 Alkarrekin lan. Irrégulier. automne 1997 - févr. 1998 (n¹-3). oct. 1999 - déc. 2001 (n4-11) [?]. - Baiona, $30 \times 21 \mathrm{~cm}$

Titre français : Ensemble. - En basque et français. - Publ. par : Eusko alkartasuna Iparralde [Bayonne]. - Suite de : Alkarrekin. - D'octobre 1999 à juil. 2001 contenu dans: Alkartasuna [Donostia]. - En nov./déc. 2004 devient : Zubiac

58 Alkarteen kaseta Züberoatik.15 déc. $1997\left(n^{\circ} 1\right)$. - Menditte, $30 \times 21 \mathrm{~cm}$ En basque; pour l'éd. française voir Lettre (La) des associations en Soule. - Publ. par : Uhaitza [Menditte]

59 Allez les noirs. Bulletin du Boucau-Tarnos-stade. 3 puis $2 n^{\circ}$ par an puis annuel. août 1990 (n $\left.{ }^{\circ} 1\right) \rightarrow$. Boucau, $30 \times 21 \mathrm{~cm}$

En français

60 Allo Ancre...! Ici Dragon...! Bulletin de liaison de la Demi-brigade coloniale de commandos parachutistes [puis de la Brigade de parachutistes coloniaux]. Mensuel. sept. oct. 1948 (nA-B). nov./déc. 1948 - juil./août 1952 (n¹-25). févr. 1954 - nov./déc. 1958 (nº 26-69). - Vannes puis Bayonne, de $27 \times 21$ à $44 \times 28 \mathrm{~cm}$

En sept. et oct. 1948 a paru sous le titre : Bulletin. Brigade coloniale commandos parachutistes S.A.S. - En français. - Devient : Béret rouge

61 Alma. Amérique latine. Lettres, terres, arts, hommes, sciences. Trimestriel. janv./mars juil./déc. 2001 ( $\left.\mathrm{n}^{\circ} 1-3\right)$. - Biarritz puis Anglet, $28 \times 22$ puis $22 \times 17 \mathrm{~cm}$

Avec un $n^{\circ} 0$ d'oct./déc. 2000. - En français; quelques textes espagnols. - Le $n^{\circ} 3$ est un $n^{\circ}$ spécial intitulé : L’Amérique latine en France. Itinéraires cachés, par Mona Huerta

62 Almanach (L') du Basque. Annuel. $1999 \rightarrow$. Romorantin, 27/29 $\times 20 \mathrm{~cm}$

En français. - Paraît dans la collection: Almanachs (Les) des terroirs de France; les différents almanachs ont un texte en partie identique

63 Alternatibaz. Errespetutsuak eta solidarioak diren praktika alternatiboen hilabetekaria. Mensuel des pratiques alternatives, respectueuses et solidaires. juil. $2004\left(\mathrm{n}^{\circ} 1\right) \rightarrow$. Kanbo, $30 \times 21 \mathrm{~cm}$ En basque et français. - Publ. par : Alternatiba [Cambo-les-Bains]

64 Alternatif. Fanzine d'expression libre et d'infos. [févr. 1988] (n¹) [?]. - Bayonne, $30 \times 21 \mathrm{~cm}$

Bulletin politique en forme de «fanzine». - En français. - Remplace : Basta [?]

Alternative ( $\left.L^{\prime}\right)$. Revue tauromachique [puis Revue alternativement tauromachique]. [1977 - 1979] (n¹-6). - Bègles puis Anglet, $30 \times 21 \mathrm{~cm}$ Avec un $n^{\circ} 0$ de [1977]. - En français. - Publ. par : Alternative (L') [Bègles]

66 Alternative ( $\left.L^{\prime}\right)$ pour Bayonne. nov. $2000\left(n^{\circ} 1\right)$. - Bayonne, $30 \times 21$ et $21 \times 15 \mathrm{~cm}$ Titre basque : Baiona hiria nagusia. - En basque et français. - Publ. dans la perspective des élections municipales de mars 2001 pour soutenir la liste «L'Alternative pour Bayonne». - A donné lieu à 2 tirages de format différent. - Remplacé par : Joalduna

67 Alternative (L') pour Bayonne [2002] voir Baiona berria Altxa! mutillak. Itsas gazteriaren aldizkaria. Le magazine des jeunes pêcheurs basques. 
Semestriel puis irrégulier. 1er sem. $1992 \rightarrow$. Ciboure puis Urrugne, $30 \times 21 \mathrm{~cm}$

En français; quelques textes basques. - Publ. par : Itsas gazteria [Ciboure puis Urrugne]. Le $n^{\circ} 7$, de 2001, est un $n^{\circ}$ spécial, de format $21 \times 15 \mathrm{~cm}$, intitulé «Les Poissons alertent les humains»; il a donné lieu à une 2e éd. en janv. 2002

Altxagarri. Le bulletin de liaison de la Vie montante en Pays basque [puis La Vie montante, Mouvement chrétien des retraités en Pays basque et sur la côte]. Trimestriel. juil. $1978\left(\mathrm{n}^{\circ} 1\right) \rightarrow$. Bayonne puis Biarritz, 29/32 $\times 21 \mathrm{~cm}$

A partir d'avr. 1980 titre français : Levain (Le). - Le sous-titre varie. - En français

Altxa-lili [puis Altxalili]. Elkartearen agerkaria, le journal de l'association [puis Altxalili elkartearen agerkaria]. Bimestriel puis irrégulier. juin 1991 - déc. 1993 (n¹-12). - Ascain, $30 \times 21 \mathrm{~cm}$

Avec un n'spécial de janv. 1993. - En français; quelques textes basques

Amaia. Bimestriel. [1969] - mai 1970 (n¹-7). - Bayonne, $27 \times 21 \mathrm{~cm}$

En français. - Dir. publ. Lucien Etchezaharreta

71 * Amaia. déc. $1982\left(n^{\circ} 1\right)$ - 1983. - [Paris], $21 \times 15 \mathrm{~cm}$

En basque et français. - Publ. par : Sustraiak-erroak [Paris]. - Contient : Jakinbideak

72

* Amarant'info. $3 n^{\circ}$ par an. [févr./mars] $2002\left(n^{\circ} 1\right) \rightarrow$. Bayonne, $30 \times 21 \mathrm{~cm}$

En français. - Publ. par : Amarante [Bayonne]

$73{ }^{*}$ Amenduze-Unaso. Amendeuix-Oneix. Bulletin municipal [ ou Informations municipales]. Annuel. $1996 \rightarrow$. Amendeuix-Oneix, $21 \times 15 \mathrm{~cm}$

En français. - Suite de : Landazaharetik Pekoberhorat

74 Amicale des anciens marins et marins anciens combattants de Bayonne voir A.M.M.A.C. Bayonne

75 Amicale des Basques de Pau et des environs «Lagunt eta maita». Bulletin de liaison [ puis Revue trimestrielle]. Trimestriel. janv. 1968 - sept. 2003 ( $n^{\circ} 1$ 144). - Pau puis Jurançon, $27 \times 21$ puis $30 \times 21 \mathrm{~cm}$

Partie du titre en gros caractères: Lagunt eta maita. - Le sous-titre varie et disparaît. - En français; quelques textes basques. - Devient : Ixtorio

76 Amicale des marins et anciens marins d'Anglet. La Gazette des marins voir Gazette (La) des marins

77 Amicale des Pieds-noirs de la Côte basque. Bulletin d'information voir Echo (L') des Pieds-noirs de la Côte basque

$78{ }^{*}$ Amicale des représentants du Pays Basque. Bulletin d'information. $1^{\mathrm{er}}$ trim. 1981 (n

${ }^{\circ}$ 1) [?]. - Biarritz, $31 \times 22 \mathrm{~cm}$

En français

79 Amicale [puis Amicale nationale] des transmissions aéroportées. Annuel ou irrégulier. [1982] $\left(\mathrm{n}^{\circ} 1\right) \rightarrow$. Bayonne, $30 \times 21 \mathrm{~cm}$

En français

80 Amikutzu. Bulletin d'informations locales trimestriel [puis Bulletin trimestriel d'informations locales]. févr. $1999\left(\mathrm{n}^{\circ} 1\right) \rightarrow$. Donapaleu, $30 \times 21 \mathrm{~cm}$ En français; quelques textes basques. - Publ. par : Amikutzu [Saint-Palais]

81 Amis (Les) de la Vieille Navarre. \{Bulletin\}. Irrégulier puis annuel. 1965 - 1971 (n¹-2). $1988\left(\mathrm{n}^{\circ} 1\right) \rightarrow$. Saint-Jean-Pied-de-Port, $24 \times 16$ puis $21 \times 15$ puis $30 \times 21 \mathrm{~cm}$ 
En 1965 et 1971 titre de départ : Association des «Amis de la Vieille Navarre». Bulletin. - En français

Amis (Les) du Bois Guilhou. Irrégulier. sept. 1996 - janv. 1998 (n¹-3). janv. $2001\left(\mathrm{n}^{\circ} 1\right) \rightarrow$. Boucau, $30 \times 21 \mathrm{~cm}$

En français. - Publ. par : Amis (Les) du Bois Guilhou [Boucau]

Amis du théâtre [puis du théâtre de la Côte basque]. \{Bulletin des Amis du théâtre de la Côte basque\}. saison 1975/76 - saison 1988/89 [?]. - Biarritz ou Bayonne, $30 \times 21$ puis $21 \times 10 / 12$ puis $21 \times 15 \mathrm{~cm}$

Avec un suppl. de 1986 intitulé : 1956 - 1986. Amis du théâtre de la Côte basque. 20 ans de théâtre. - En 1975/76 a paru sous le titre : Bulletin des Amis du théâtre de la Côte basque. En français. - Paraît durant la saison théâtrale (jusqu'à $7 n^{\circ}$ par saison)

Amis du théâtre de la Côte basque. \{Saison\} [ou Programme]. Annuel. [...] $\rightarrow$. Biarritz ou Bayonne, de $21 \times 11$ à $30 \times 21$ ou $23 \times 23$ ou $15 \times 15 \mathrm{~cm}$

Paraissait déjà en 1974/75. - En français

Amodioren izarra. D.Z. gaztetxeko zine. [1991] (n¹, «zpezial de l'été»). - Saint-Jean-deLuz, $30 \times 21 \mathrm{~cm}$

Bulletin en forme de «fanzine». - En français; quelques textes basques. - Publ. par : DonibaneZiburuko gaztetxea [Saint-Jean-de-Luz]

Anacoluthe. Ex-L'Heureux né Cassin. 1984 (n¹)-[...] - Bayonne, 30 x $21 \mathrm{~cm}$

Paraissait encore en 1985/86. - En français - Rédigé par des élèves du lycée René Cassin [Bayonne]

Angelu! Angeluko abertzaleen aldizkaria. Le journal d'Anglet-Euskal herri. mai 1996 [sept. 1999] (n¹-2). - Anglet, $30 \times 21$ puis $21 \times 15 \mathrm{~cm}$

En basque et français. - Publ. par : Angelu zain [Anglet]

Anglet. Revue municipale d'information [puis Bulletin officiel municipal puis Bulletin municipal officiel puis Bulletin municipal d'information]. Annuel puis bimestriel. [1964] janv. 1977 (n¹-14). 1978 - 1988. mai 1989 - févr. 1991 (n¹-10). - Anglet, Paris puis Anglet, Fontenay-le-Comte puis Anglet, $27 \times 19$ puis $30 \times 21 \mathrm{~cm}$

Avec un n'spécial de [1989] intitulé : Anglet. Bilan d'un mandat. - Titre de départ de 1965 à 1970 : Revue municipale d'information de la Ville d'Anglet, puis de 1971 à 1977 : Bulletin officiel municipal \{d'\} Anglet. - En français. - De 1978 à 1984 paraît parallèlement à : Anglet. La lettre municipale, puis en 1988 à : Lettre (La) d'Anglet. - Devient : Anglet-magazine

Anglet. La lettre municipale. \{Bulletin trimestriel\}. Trimestriel ou irrégulier. mars 1979 oct. 1984 (n¹-11). - Anglet, $28 \times 19$ puis $30 \times 21 \mathrm{~cm}$

En français. - Paraît parallèlement à: Anglet. Revue municipale... - En 1988 devient : Lettre (La) d'Anglet

Anglet-avenir. Réfléchir, informer, proposer, agir. Bimestriel. janv. - nov. 1987 ( $\left.n^{\circ} 1-5\right)$. janv. - mars 1989 (n6-7). - Anglet, $30 \times 21 \mathrm{~cm}$ En français. - Publ. par : Anglet-avenir [Anglet] Anglet-Côte basque-basket... voir A.C.B.B...

92 Anglet-Hormadi club. \{Hockey sur glace. Saison\}... Annuel. [...] - 1991/92. 1997/98. Anglet, $30 \times 21 \mathrm{~cm}$

Paraissait déjà en 1979/80; n'a pas paru tous les ans. - En français. - Remplacé par : Magazine (Le) de l'Hormadi

93 Anglet-magazine. \{Journal d'informations municipales [puis Journal municipal d'information] de la Ville d'Anglet\}. Bimestriel. avr. $1991\left(\mathrm{n}^{\circ} 11\right) \rightarrow$. Anglet, $30 \times 21$ puis 
$30 \times 23 \mathrm{~cm}$

D'avr. 2001 à sept./oct. 2004 a paru sous le titre : Anglet. Magazine de la ville. - En français. Suite de : Anglet. Revue municipale... juin $2004 \rightarrow$. Anglet, $30 \times 21 \mathrm{~cm}$ En français

95 Anglet olympique. Club omnisports. [...] - 2002. - Anglet, $30 \times 21 \mathrm{~cm}$ En français Anglet olympique. Rugby [puis Anglet olympique rugby club].\{Saison\}. Annuel. 1993/94 1994/95. 1996/97 $\rightarrow$. Anglet, $30 \times 21 \mathrm{~cm}$ En français

\section{$30 \times 21 \mathrm{~cm}$}

En français 
115 Arin-foot. Bulletin d'information de l'Arin luzien. sept. 1990 (n¹) - [...]. - Saint-Jean-deLuz, $30 \times 21 \mathrm{~cm}$ En français

116 Arin luzien. Saison. Annuel. 1986/87 [?] $\rightarrow$. Saint-Jean-de-Luz, $30 \times 21 \mathrm{~cm}$ En français

117 Armanac \{capitou\} publicat per l'Académie gascoune de Bayoune voir Académie gascoune, Bayoune. Armanac capitou

118 Arradoy xolan. Bulletin municipal. Annuel. $1983 \rightarrow$. Ispoure, $30 \times 21 \mathrm{~cm}$ En français

119 Arraia. Journal édité par Geroa zain. Geroa zainen aldizkaria. avr. 1990 - janv. 1992 (n 1-4). - Hazparne, $30 \times 21 \mathrm{~cm}$

Avec un $n^{\circ}$ spécial de [févr. 1998] publ. à l'occasion des élections cantonales de mars 1998 pour soutenir la candidature de Xarlo Etxezaharreta. - En français; quelques textes basques

Arrangoitzeko berriak. Semestriel. déc. $1983\left(n^{\circ} 1\right) \rightarrow$. Arcangues, $30 \times 21 \mathrm{~cm}$ Bulletin municipal d'Arcangues. - En français

121 Arrapitz lerroz lerro. \{L'actualité et la vie du réseau\}. Irrégulier. juil. 2002 - juin 2003 (n 1-3). juil. $2004\left(\mathrm{n}^{\circ} 4\right) \rightarrow$. Saint-Palais, $30 \times 21 \mathrm{~cm}$

Partie du titre en gros caractères: Lerroz Lerro. - En français; quelques textes basques. - Publ. par : Fédération pour un nouveau développement rural Arrapitz [Saint-Palais]. - En sept. 2004 absorbe provisoirement :B.L.E. berri

* Arroka. Lokarria. Irrégulier. nov. $2004\left(n^{\circ} 1\right) \rightarrow$. Biarritz, $30 \times 21 \mathrm{~cm}$

En français; quelques textes basques. - Publ. par : Arroka [Biarritz]

Art. 2. Unir, animer, défendre. La lettre de l'Union commerciale de Bayonne. Mensuel ou

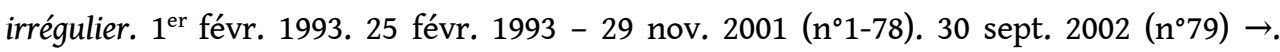
Bayonne, $30 \times 21$ puis $21 \times 15 \mathrm{~cm}$ Avec un $n^{\circ} 0$ du 28 oct. 1992 et plusieurs nspéciaux. - De juin à nov. 2001 (n76-78) a paru sous le titre: Article 2 : Unir, animer, défendre. - En français. - Publ. par : Union commerciale et artisanale de Bayonne [Bayonne] 
124 Artha. Attention et vigilance. Journal de l'association L'Artha. Irrégulier. mars 1996 juin 2000 ( $\left.\mathrm{n}^{\circ} 1-11\right)$. - Saint-Jean-de-Luz, $30 \times 21 \mathrm{~cm}$ En français

Artha (L'). Bulletin de la Section de Saint-Jean-de-Luz \{-Ciboure\} du Parti communiste français [puis Journal de la Liste de rassemblement des forces de gauche et de progrès soutenue par le P.C.F.]. Irrégulier. août 1952 - [mars 1989] [?]. - [Saint-Jean-de-Luz], de $27 \times 21 \grave{a} 42 \times 29 \mathrm{~cm}$

Le sous-titre varie. - En français. - Publ. notamment à l'occasion de diverses élections $1982\left(n^{\circ} 1-3\right)$. - Urcuit, $30 \times 21 \mathrm{~cm}$ Le n'2 a paru sous le titre: Bulletin d'information de l'Association de propriétaires d'Urcuit; le $n^{\circ} 3$ a paru sous le titre : Bulletin de l'Association... - En français 
141 Association des maires du Pays basque. Bulletin de liaison. 22 déc. 1993 ( $n^{\circ} 1$ ). Hélette, Ascain, $30 \times 21 \mathrm{~cm}$

Titre basque : Euskal herriko auzapezen batasuna. Gure agerkaria. - En français

Association des naturalistes du Pays basque. La Soldanella villosa voir Soldanella (La) villosa

Association des paralysés de France. Délégation des Basses-Pyrénées voir Lien A.P.F.

Association des parents d'élèves du Lycée et du C.E.S. de Bayonne-Marracq. Bulletin de liaison. févr. 1969 - mai 1970 (nº 1-5 [sic pour 4]). - [Bayonne], $27 \times 21 \mathrm{~cm}$

En français. - Devient : Echo (L') des parents

145 Association des usagers du Centre social Polo-Beyris. Bulletin de liaison. $2 n^{\circ}$ par an. 16 déc. $1991\left(n^{\circ} 1\right)$ - juin $1995\left(n^{\circ} 2\right)$. - [Bayonne], $30 \times 21 \mathrm{~cm}$

En français. - Eléments repris par : P'tit (Le) bavard

$146 *$ Association «Gure kondaira». $1978\left(\mathrm{n}^{\circ} 1\right)$ [?]. - Bayonne, $30 \times 21 \mathrm{~cm}$

En français

147 Association nationale des membres de l'Ordre national du mérite. Annuaire de la Section des Pyrénées-Atlantiques. Irrégulier. $1987 \rightarrow$. Billère puis Anglet puis Pau, $21 \times 15 \mathrm{~cm}$

En français

148 Association pour la recherche et l'information sur les toxicomanies... voir A.R.I.T.info

149 Association pour la recherche spéléologique internationale... voir A.R.S.I.P.

150 Association Qui ose gagne \{et\} Anciens du 6. Annuaire. Irrégulier. $1999 \rightarrow$. Bayonne, $21 \times 30 \mathrm{~cm}$

En français

151 Association Qui ose gagne \{et\} Anciens du 6. Bulletin d'information [puis Bulletin de liaison]. $3 n^{\circ}$ par an. 24 mars $1999\left(\mathrm{n}^{\circ} 1\right) \rightarrow$. Bayonne, $30 \times 21 \mathrm{~cm}$ En français

152 Association sportive bayonnaise. Saison... Rugby, karaté, triathlon. Annuel. [...] $\rightarrow$. Bayonne, $27 \times 21$ puis $30 \times 21 \mathrm{~cm}$

Paraissait déjà en 1972/73. - Jusqu'en 1989/90 a paru sous le titre : A.S.B. Panorama sportif [ou Saison]... - Le sous-titre varie et disparait. - En français

153 Association «Villefranque cadre de vie». Bulletin d'information. [nov. 1998 - avr. $\left.2000\left(n^{\circ} 1-4\right)\right]$. - [Villefranque], $30 \times 21 \mathrm{~cm}$

En français

154 Astapito. Irrégulier. [oct./nov. 2001] $\rightarrow$. [Mauléon], $30 \times 21 \mathrm{~cm}$

Bulletin politique en forme de "fanzine».. - En français; quelques textes basques

$155 *$ Astintzen. [...] $\rightarrow$. Bayonne [?], $21 \times 15 \mathrm{~cm}$

Paraissait en 2003 et 2004. - Publ. par : Segi

156 Astrochoc. Journal trimestriel d'informations astrologiques. sept. $1987\left(\mathrm{n}^{\circ} 1\right)$ [?]. - Anglet, $30 \times 21 \mathrm{~cm}$

En français

157 Atalante (L')-cinéma [puis Atalante (L'). Cinéma V.O., resto, expos. Gazette]. Toutes les 3 semaines puis mensuel. 19 sept./20 oct. $1990\left(\mathrm{n}^{\circ} 1\right) \rightarrow$. Bayonne, $30 \times 21$ puis $25 \times 18 \mathrm{~cm}$ 
En français. - Publ. par : Association «Cinéma et cultures» [Bayonne]. - Remplace : Utopiacinéma

Atalaya. La revue trimestrielle de l'histoire, du patrimoine et de l'architecture de Biarritz. juin $2003\left(n^{\circ} 1\right) \rightarrow$. Biarritz, $30 \times 21 \mathrm{~cm}$

En français. - Publ. par : Association des amis de la Chapelle impériale et du patrimoine de Biarritz [Biarritz]

Atchiki. Edition du Pays basque. Organe \{mensuel\} de l'Association départementale des combattants prisonniers de guerre des Basses-Pyrénées [puis des combattants prisonniers de guerre et C.A.T.M. [combattants d'Algérie-Tunisie-Maroc] des Pyrénées-Atlantiques]. Mensuel ou irrégulier. 1948 [?] $\rightarrow$. Bayonne, $45 \times 30$ puis $59 \times 43$ puis $43 \times 30 \mathrm{~cm}$

Titre béarnais: Units toustem; titre français: Tenir. - Le sous-titre varie. - En français. - A partir de 1965 constitué en grande partie par le texte du journal national de la Fédération nationale des combattants prisonniers de guerre : P.G. (Le) qui devient : P.G.-C.A.T.M. (Le) [Paris]; à partir de déc. 1972 porte au-dessus du titre : Le P.G. et Atchiki réunis. - Une éd. Béarn-Pays de Soule a paru parallèlement à Pau sous le même titre

160 Ateka. Ezker abertzaleen ilabetekari. Mensuel. oct. 1983 - mars 1987 (n¹-40). - Bayonne, $30 \times 21 \mathrm{~cm}$

Avec un prospectus de [1983] et un no 0 d'août 1983. - En basque et français. - En mai 1986 certains éléments repris par : Ekaitza

161 Atera. Euskal zerbitzuen aldizkaria. Uda leku eta irakasleen arteko har emana. [janv. 1998] (nº) [?]. - [Bayonne], $30 \times 21 \mathrm{~cm}$

En basque. - Publ. par: Uda leku elkartea [Bayonne]. - En juin 2004 éléments repris par: Berripapera

Atlantica. Aquitaine-Euskadi [puis Aquitaine-Euskadi-Navarre]. Revue transfrontalière d'information régionale et européenne. Bimestriel. mai 1991 - oct./déc. 1994 (n¹-19). Biarritz, $30 \times 21 \mathrm{~cm}$

Porte au-dessus du titre : Euromagazine. - En français. - Une éd. espagnole a paru à Bilbao. Pour les suppl. voir les notices suivantes

163 Atlantica. Revue transfrontalière d'information régionale et européenne. Supplément économique. sept. 1992. - Biarritz, $30 \times 21 \mathrm{~cm}$ En français

164 Atlantica. Les idées, les lettres et arts, les hommes atlantiques. Supplément littéraire du magazine... Irrégulier. nov. 1991 - [janv. 1994] $\left(12 \mathrm{n}^{\circ}\right)$. - Biarritz $30 \times 21 \mathrm{~cm}$ Les premiers $n^{\circ}$ portent au dessus du titre: Eurorevue. - En français. - Devient: Atlantica littéraire

Atlantica littéraire. Les idées, les lettres et arts, les hommes atlantiques. Mensuel. mars 1994 - déc. 1997 ( $\left.n^{\circ} 1-47\right)$. - Biarritz, $30 \times 21 \mathrm{~cm}$ Avec des suppl. au $n^{\circ} 17$ et 18 (juin et juil. 1995). - En français. - Suite de: Atlantica... Supplément littéraire... - Devient : Atlantica-magazine

166 Atlantica-magazine. Les lettres, les arts, les hommes, les terres atlantiques. Mensuel. janv. $1998\left(\mathrm{n}^{\circ} 48\right) \rightarrow$. Biarritz puis Anglet, $30 \times 21 \mathrm{~cm}$ Avec de nombreux suppl. et $n^{\circ}$ spéciaux. - En français. - Suite de : Atlantica littéraire

167 * Atlantique-Pyrénées. Côte basque-magazine. Supplément de «La Voix du Pays basque». \{Organe du Rassemblement «Pays Basque»\}. 1974 - 1977 [?]. - Biarritz, Bayonne puis 
Biarritz, 30/32 $\times 21 \mathrm{~cm}$

En français. - Dir. publ. Georges de Styn. - Suite de : Côte basque-magazine [1966 - 1973]

Atzar. [mars 1990] (n¹). mars $1991\left(\mathrm{n}^{\circ} 1\right)$ [?]. - Ziburu, $30 \times 21 \mathrm{~cm}$

En basque et français. - Publ. par: Gazte abertzaleen batasun euskalduna (G.A.B.E.) [Ciboure]; voir aussi Zirta

Au bout du fil. Journal d'information de La Cotonnière basquaise, filature et retorderie de coton à Anglet... Trimestriel. $1^{\mathrm{er}}$ janv. $-1^{\mathrm{er}}$ juil. $1962\left(\mathrm{n}^{\circ} 1-3\right)$ [?]. - Anglet, $27 \times 21 \mathrm{~cm}$ En français

$170 *$ Au pas... au trot... au galop! Trimestriel puis bimestriel [?]. [juil./sept. 1996] - sept./oct. 1997 (n¹-7). - Biarritz, $30 \times 21 \mathrm{~cm}$

En français. - Publ. par : Club hippique d'Ilbarritz [Biarritz]

171 Au pays des rêves. printemps $1979\left(\mathrm{n}^{\circ} 1\right)$ [?]. - Anglet, $15 \times 21 \mathrm{~cm}$ Recueil de dessins d'élèves de l'Ecole Jules Ferry (Anglet), impr. par l'Imprimerie spéciale de La Ronde. - En français

172 Au pied de l'Ursuya. Journal des enfants de la classe de perfectionnement, Groupe scolaire Jean Verdun, Hasparren. sept. 1986 - 1987/88. - Hasparren, $30 \times 21 \mathrm{~cm}$

Avec un $n^{\circ}$ spécial du 1er trim. de l'année scolaire 1985/86, composé de dessins d'élèves, impr. par l'Imprimerie spéciale de La Ronde. - En français

173 Au service de tous voir Denen zerbitzari et Zerbitzari

Au soleil de la saison voir Denak argian et Lumière sur l'Adour

Aujourd'hui et demain voir Egun eta bihar

* Aumo-magazine. [1988/89] - [...]. - Saint-Jean-de-Luz, $30 \times 21 \mathrm{~cm}$ En français. - Publ. par : Aumônerie du Collège Maurice Ravel [Saint-Jean-de-Luz]

177 Aupa Ciboure. Bulletin diffusé par l'association Aupa Ciboure. janv. 1998 - févr. 2001 (5 n '). - Ciboure, $30 \times 21 \mathrm{~cm}$ Titre basque : Aupa Ziburu. - En français

$178 *$ Auto-occasion. Bimensuel. 1988-1989 [?]. - Ondres puis Biarritz, $30 \times 23$ puis $41 \times 29 \mathrm{~cm}$ En français

179 Autour du clocher [ou du clocher de Mauléon]. Bulletin paroissial de Mauléon [puis Bulletin mensuel interparoissial de la Soule]. Mensuel. avr. 1910 ( $1^{\text {re }}$ année, $\left.\mathrm{n}^{\circ} 1\right)-[\ldots]$. janv. 1956 - déc. 1959 ( $\left.n^{\circ} 1-41\right)$. janv. $1960 \rightarrow$. Mauléon, $22 \times 14$ puis $38 \times 29 \mathrm{~cm}$

A partir de mai 1979 titre basque : Zeiñützülian. - En français; à partir de 1960 quelques textes espagnols ou basques

Autrement. Publication de l'association «Boucau autrement» des femmes et des hommes de gauche et de progrès. Irrégulier. févr. 1996 - nov. 1998 (n¹-7). - Boucau, $30 \times 21 \mathrm{~cm}$ En français. - En 2000 certains éléments repris par : Gauche (La) plurielle Boucau

181 Avec... l’amicale laïque Adixkideak. Bulletin. Irrégulier. [déc. $1994\left(n^{\circ} 1\right)$ ] $\rightarrow$. Urrugne, 30 $21 \mathrm{~cm}$ Avec plusieurs suppl. - En français. - Publ. par : Adixkideak [Urrugne]

182 * Avenir (L'). Journal de la Cellule Catelas du P.C.F. Rue Maubec, Tourettes, Bedat, Citadelle. [...]. - [Bayonne], $27 \times 21 \mathrm{~cm}$

Paraissait en 1971. - En français. - Publ. par : Parti communiste français. Cellule Catelas [Bayonne] 
* Avenir (L'). Pour une démocratie avancée vers le socialisme. Journal de Bayonne-Est du Parti communiste français. Bayonne-rive droite. [...]. - [Bayonne], $34 \times 27 \mathrm{~cm}$ Paraissait en 1971 et 1973. - En français Avenir (L') d'Ascain. 1971 (n¹) [?]. mars 1977 (n²). mars 1983 (n³-4). févr. 1989 (n5-6). juin $1995\left(\mathrm{n}^{\circ} 7\right)$. - Ascain, de $40 \times 25$ à $30 \times 21 \mathrm{~cm}$ Titre basque: Azkaine aintzina. - En français. - Publ. à l'occasion des élections municipales successives pour soutenir la liste présentée par André Luberriaga Avenir (L') du Béarn et du Pays basque. 17 déc. [1955] [?]. - Biarritz, $65 \times 46 \mathrm{~cm}$ En français. - Publ. à l'occasion des élections législatives de janv. 1956 pour soutenir la Liste des indépendants-paysans des Basses-Pyrénées. - Dir. publ. Guy Petit Aviron bayonnais, club omnisports. \{Saison\}. Annuel. [...] $\rightarrow$. Bayonne, $27 \times 21 \mathrm{~cm}$ Paraissait déjà en 1944/45; jusqu'en 1966 a paru sous le titre: A.B. Saison (format $12 \times 16 \mathrm{~cm})$. - En français. - Publ. par : Aviron bayonnais [Bayonne]

187 Aviron bayonnais football club. \{Saison\}. Annuel. 1996/97 $\rightarrow$. Bayonne, $30 \times 21 \mathrm{~cm}$ En français. - Publ. par : Aviron bayonnais football club [Bayonne] Aviron bayonnais rugby. Annuel. 2000/01 - 2002/03. - Bayonne, $30 \times 21 \mathrm{~cm}$ En français. - Publ. par : Aviron bayonnais rugby pro [Bayonne] Avis (L’) du quartier. déc. 1996 (nº). avr. 1997 (n¹). [sept. 1997] (nºspécial). - Bayonne, $30 \times 21 \mathrm{~cm}$ Le no a paru sous le titre: Journal. Centre social Pyrène. - En français. - Publ. par : Centre social Pyrène [Bayonne], puis en 1997 par : Maison de la vie citoyenne Bayonne - SainteCroix [Bayonne]

Ayherre. Bulletin municipal \{d'information\}. Annuel. [...] $\rightarrow$. Ayherre, $25 \times 18$ puis $30 \times 21 \mathrm{~cm}$

Paraissait déjà en 1986. - Certains nºnt paru sous le titre: Bulletin municipal. Ayherre. - En français

191 Azia-info. Irrégulier. sept. 1999 - août $2002\left(n^{\circ} 1-6\right)$. nov. $2004\left(n^{\circ} 7\right) \rightarrow$. Tardets, $30 \times 21 \mathrm{~cm}$ En français. - Publ. par : Azia [Tardets]

192 Azkaine. Herriko agerkari. Annuel puis irrégulier. avr. $1978\left(n^{\circ} 1\right) \rightarrow$. Ascain, $30 \times 22 \mathrm{~cm}$ Titre français: Ascain. Bulletin municipal. - En français ou en basque et français. - En 1982 parait parallèlement: Commune d'Ascain. La lettre du maire Azkaine aintzina voir Avenir (L') d'Ascain

Azkain-go herria. Berriak voir Commune d'Ascain. La lettre du maire

\section{INDEX}

Index chronologique : 20e siècle

Index géographique : Pays basque (France)

Thèmes : bibliographie

Mots-clés : publication périodique 